Supporting Information

\title{
Improved Durability of Electrocatalyst Based on Coating of Carbon Black with Polybenzimidazole and their Application in Polymer Electrolyte Fuel Cells
}

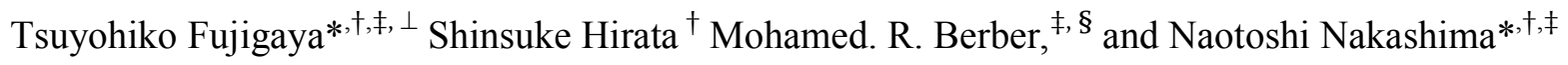

${ }^{\dagger}$ Department of Applied Chemistry, Graduate School of Engineering, Kyushu University, 744

Motooka, Nishi-ku, Fukuoka, 819-0395, Japan.

${ }^{\ddagger}$ The World Premier International Research Center Initiative, International Institute for Carbon

Neutral Energy Research (WPI-I²CNER), Kyushu University 744 Motooka, Nishi-ku, Fukuoka,

819-0395, Japan.

${ }^{\S}$ Department of Chemistry, Faculty of Science, Tanta University, Tanta 31527, Egypt

${ }^{\perp}$ JST-PRESTO, 4-1-8 Honcho, Kawaguchi, Saitama, 332-0012, Japan

*E-mail: fujigaya.tsuyohiko.948@m.kyushu-u.ac.jp

*E-mail: nakashima.naotoshi.614@m.kyushu-u.ac.jp 
Table S1. Elemental analysis of Vulcan/PBI and Ketjen/PBI.

\begin{tabular}{|c|c|c|c|c|}
\hline & $\mathrm{H}$ & $\mathrm{C}$ & $\mathrm{N}$ & $\mathrm{O}$ \\
\hline Vulcan/PBI & 0.51 & 95.17 & 1.04 & 3.28 \\
\hline Ketjen/PBI & 1.16 & 92.44 & 3.71 & 2.69 \\
\hline
\end{tabular}

Table S2. Peak analysis of C 1s narrow scan before (upper) and after (lower) of (a) Vulcan/Pt and (b) Vulcan/PBI/Pt

a (before)

\begin{tabular}{|c|c|c|c|c|}
\hline $\mathbf{C - C}$ & $\mathbf{C - O}$ & $\mathbf{C = 0}$ & $\mathbf{C O O}$ & $\mathbf{C - F}$ \\
\hline 284.2 & 285.2 & 286 & 289.4 & 291.1 \\
\hline $65.2 \%$ & $21.4 \%$ & $3.2 \%$ & $10.2 \%$ & - \\
\hline
\end{tabular}

\begin{tabular}{|c|l|l|l|l|}
\hline (after) & \multicolumn{5}{|c|}{} \\
\hline C-C & C-O & C=0 & COO & C-F \\
\hline 284.2 & 285.2 & 286 & 289.4 & 291.1 \\
\hline $49.8 \%$ & $33.7 \%$ & $3.4 \%$ & $13.1 \%$ & - \\
\hline
\end{tabular}

b (before)

\begin{tabular}{|l|l|l|l|l|}
\hline C-C & C-O & C=O & COO & C-F \\
\hline 284.2 & 285.2 & 286 & 289.4 & 291.1 \\
\hline $57.6 \%$ & $17.3 \%$ & $5.5 \%$ & $19.7 \%$ & - \\
\hline
\end{tabular}

\begin{tabular}{|l|l|l|l|l|}
\multicolumn{3}{|c|}{ (after) } \\
\hline C-C & C-O & C=0 & Coo & C-F \\
\hline 284.2 & 285.2 & 286 & 289.4 & 291.1 \\
\hline $60.8 \%$ & $6.2 \%$ & $15.2 \%$ & $17.8 \%$ & - \\
\hline
\end{tabular}
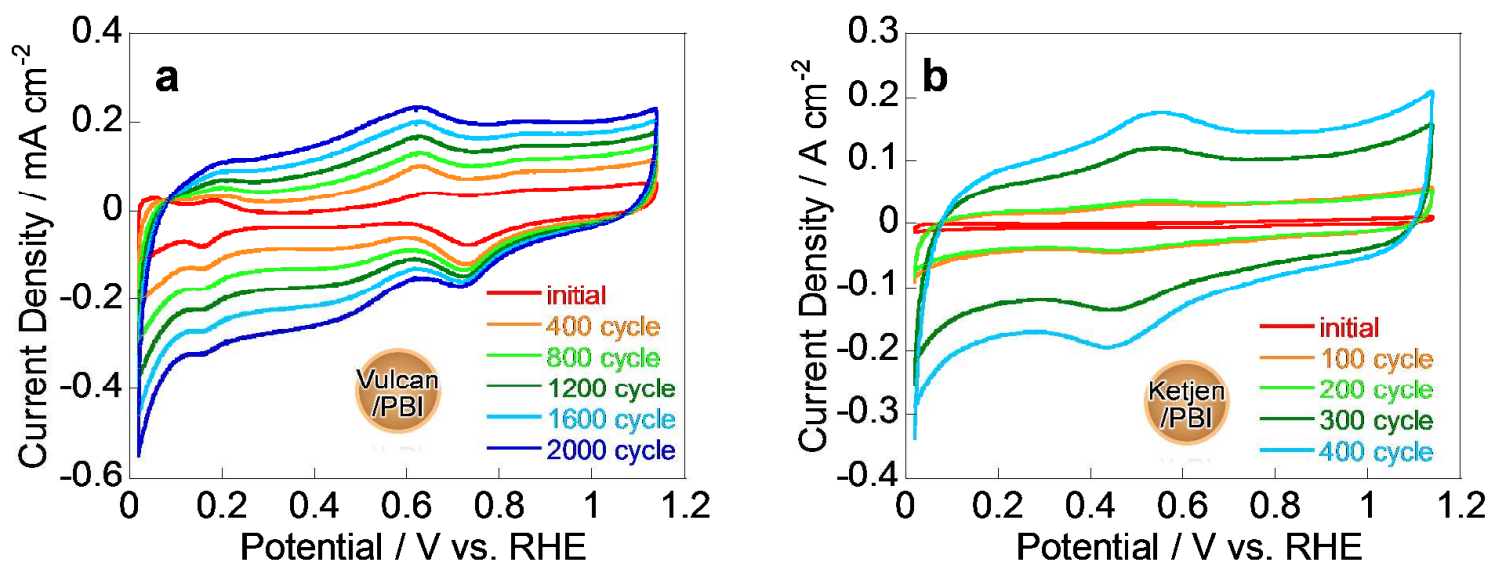

Figure S1. CVs of the (a) Vulcan/PBI and (b) Ketjen/PBI measured in $0.1 \mathrm{M} \mathrm{HClO}_{4}$ aqueous solutions, which are magnification of (a) Figure $2 \mathrm{~b}$ and (b) Figure $2 \mathrm{~d}$. 

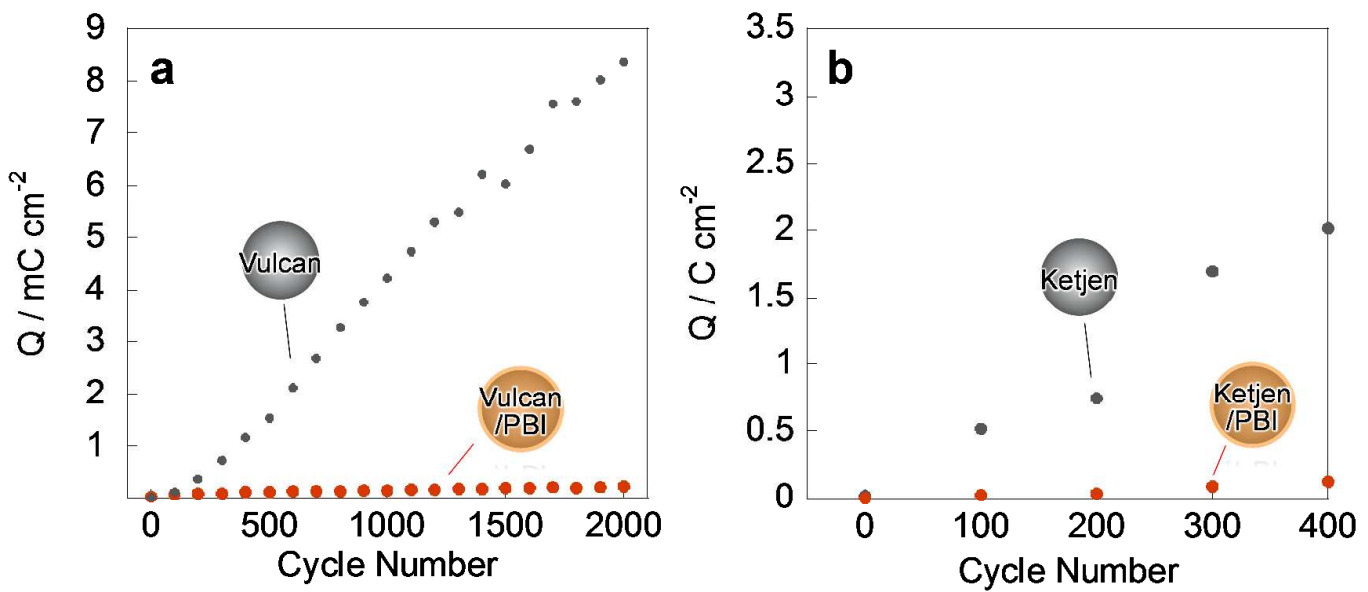

Figure S2. Plots of the area of quinone values for the (a) Vulcan (black) and Vulcan/PBI (red) and (b) Ketjen (black) and Ketjen/PBI (red) as a function of the cycle number measured in $0.1 \mathrm{M} \mathrm{HClO}_{4}$ aqueous solutions.
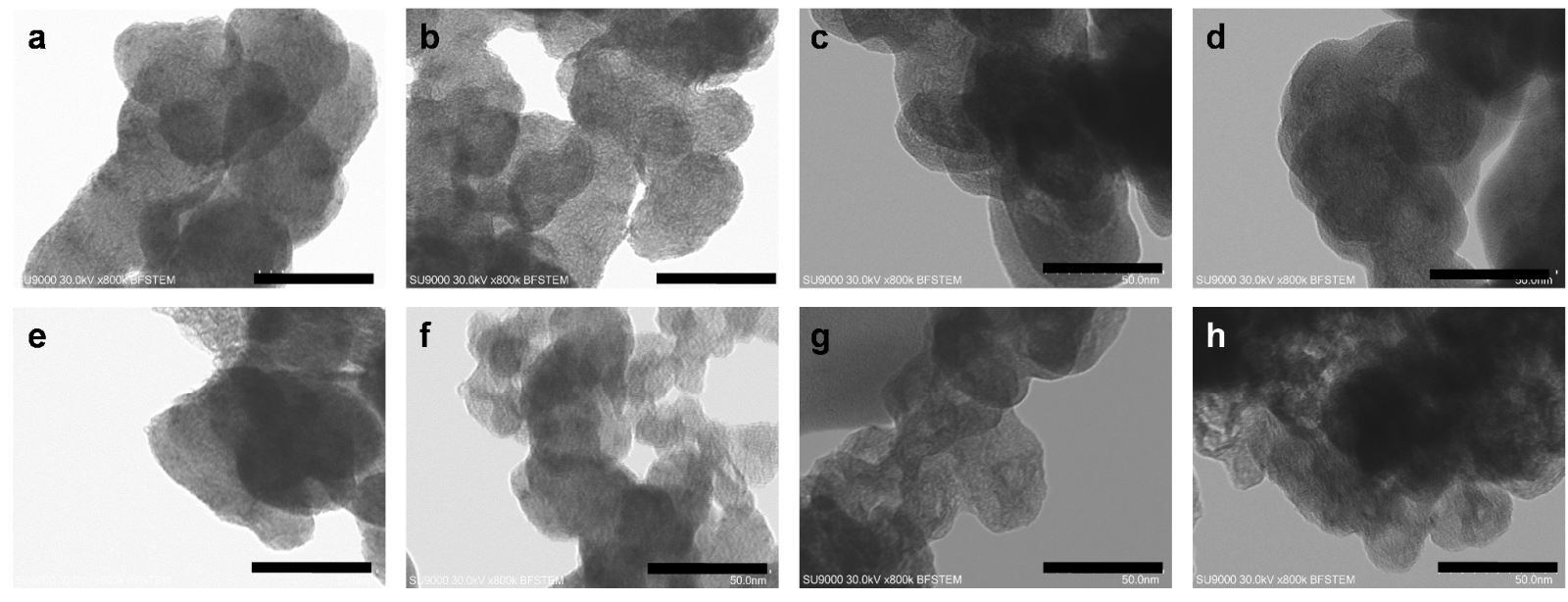

Figure S3. TEM images of (a, e) Vulcan, (b, f) Vulcan/PBI, (c, g) Ketjen and (d, h) Ketjen/PBI before (a-d) and after (e-h) the durability test. Scale bars; $50 \mathrm{~nm}$. 


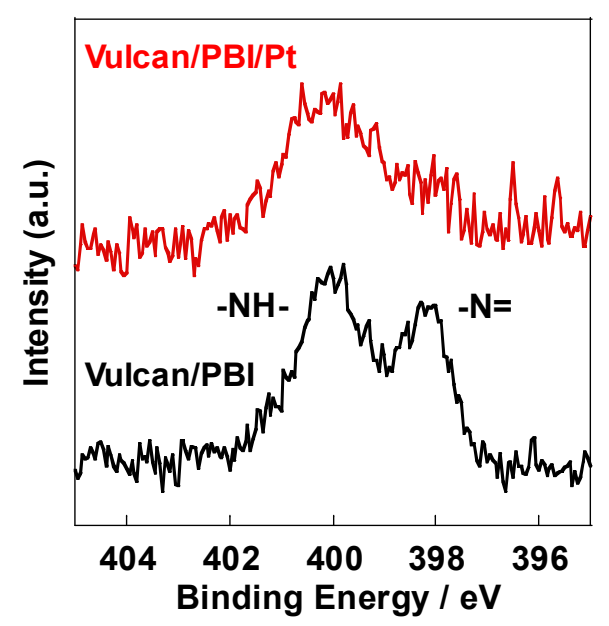

Figure S4. XPS N 1s narrow scans for Vulcan/PBI (black) and Vulcan/PBI/Pt (red). N= at $400 \mathrm{eV}$ before Pt loading was severely decreased due to the delocalization of the lone pair electron after the Pt loading. 

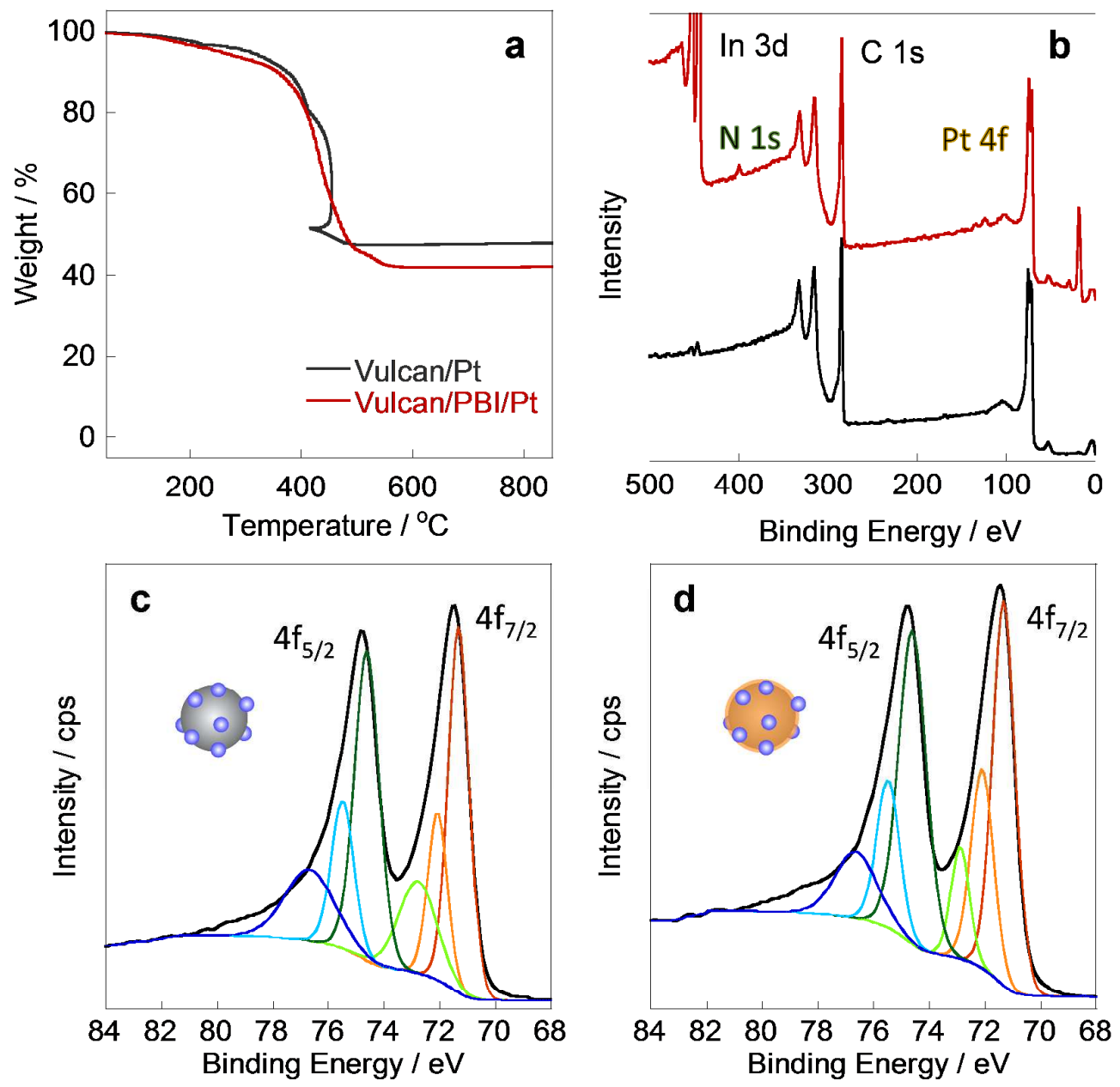

Figure S5. (a) TGA curves of the Vulcan/Pt (black) and Vulcan/PBI/Pt (red). (b) XPS survey scans of the Vulcan/Pt (black) and Vulcan/PBI/Pt (red). XPS narrow scans of (c) Vulcan/Pt (black) and (d) Vulcan/PBI/Pt (black) for the Pt 4f, in which the deconvolution curves are shown, and the results are listed in Figure 4d. 

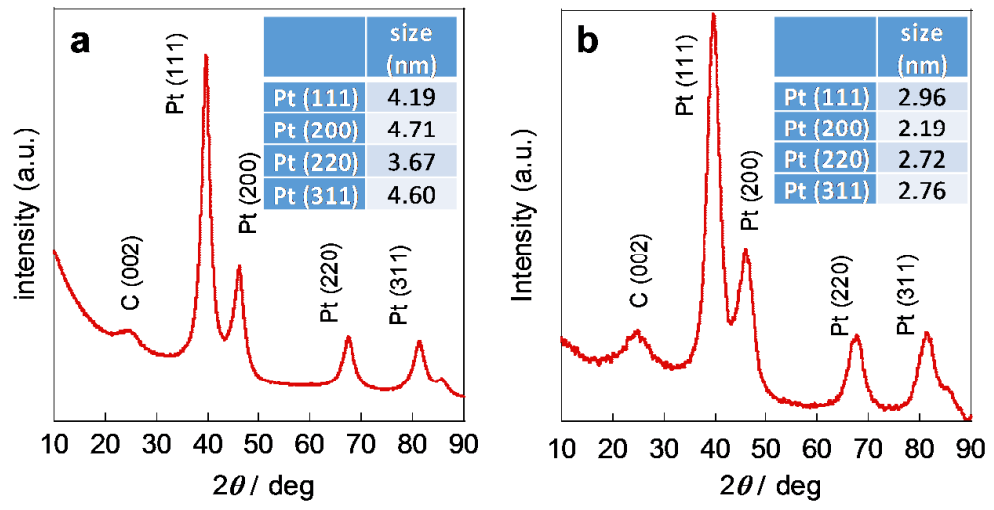

Figure S6. XRD profiles of the (a) Vulcan/Pt and (b) Vulcan/PBI/Pt. (Inset) List of the Pt domain size calculated based on Scherrer equation. ${ }^{\mathrm{S} 1}$ Larger size of $\mathrm{Pt}$ domain for Vulcan/Pt than that of TEM observation was probably caused by the presence of small number of large particles $(>10 \mathrm{~nm})$.
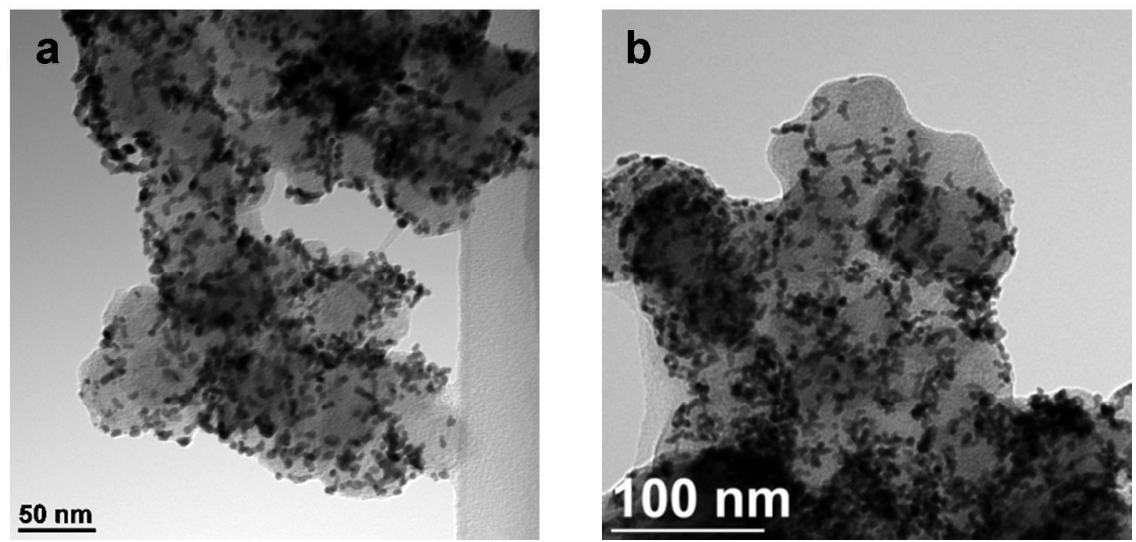

Figure S7. TEM images of (a) Vulcan/Pt and (b) Vulcan/PBI/Pt after the durability test. 

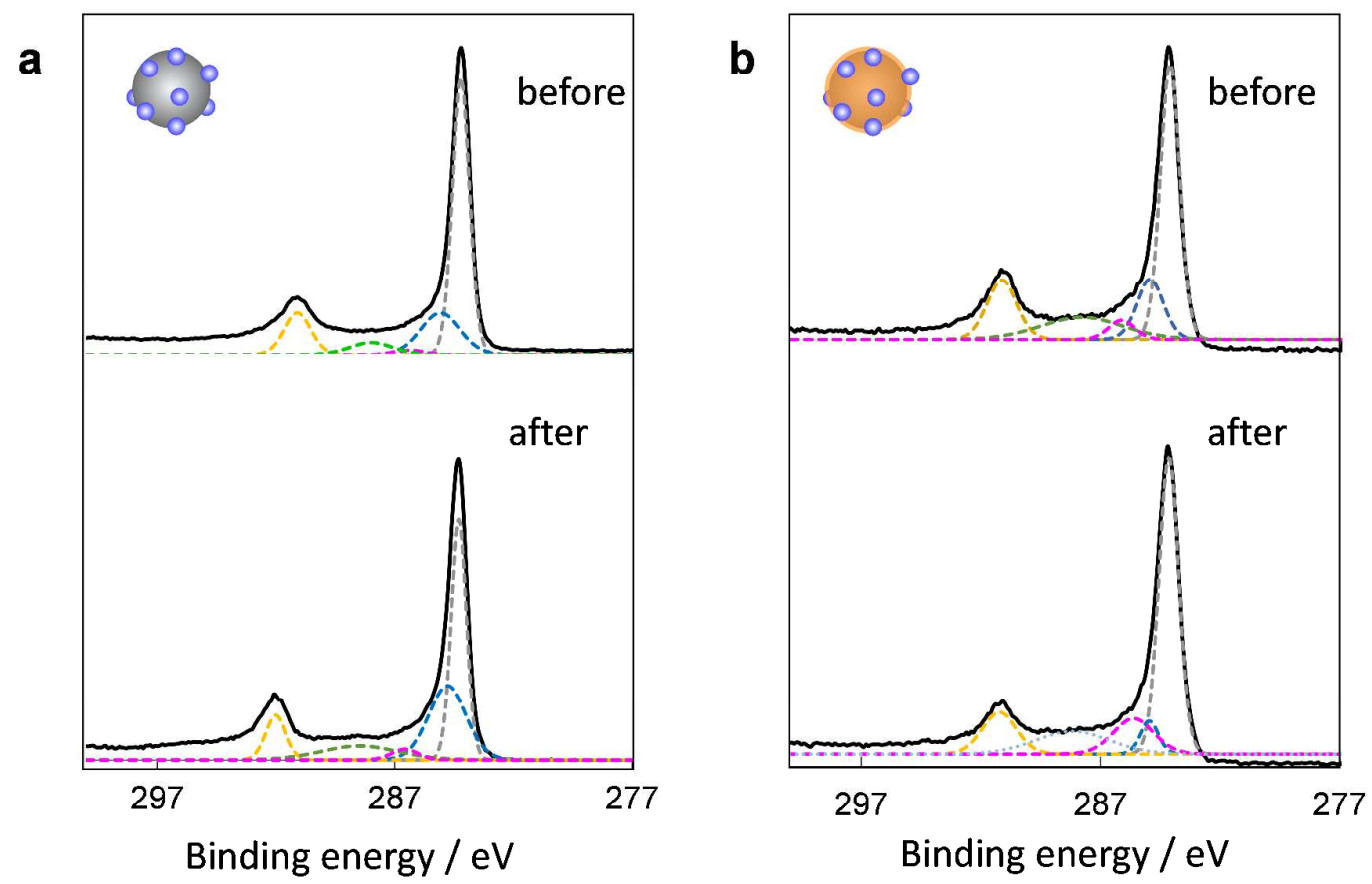

Figure S8. XPS C1s narrow scans of the (a) Nafion-MEA $\mathrm{A}_{\text {non-coat }}$ and (b) Nafion-MEA $\mathrm{A}_{\text {coat }}$ before (upper line) and after (lower line) the durability tests. Deconvolution lines for C-C (gray line), C-O (blue line), $\mathrm{C}=\mathrm{O}$ (pink line), $\mathrm{COO}$ (green line) and $\mathrm{C}-\mathrm{F}$ (orange line) were also displayed. The results are summarized in Table S2.
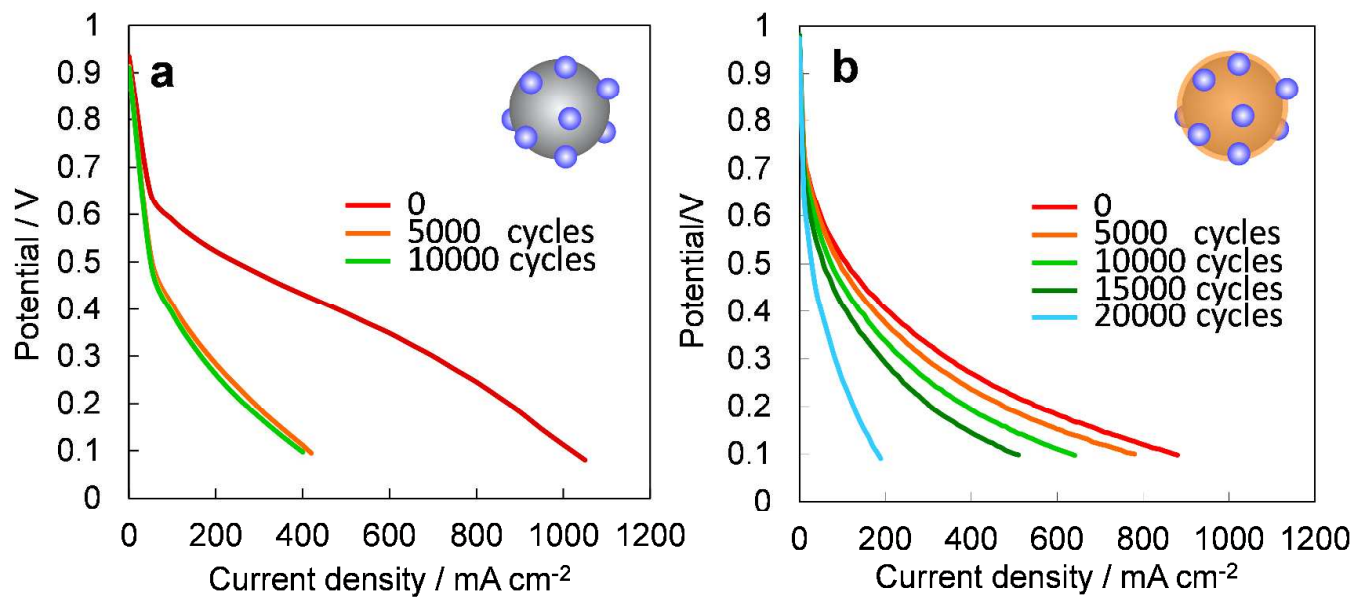

Figure S9. Polarization curves during the durability test for (a) $M_{E A} A_{\text {non-coat }}$ and (b) $M_{E} A_{\text {coat }}$, in which representative polarization curves are displayed. ${ }^{\mathrm{S} 2}$ Reprinted in part with permission from ref. S2. Copyright 2014 Royal Society of Chemistry. 


\section{Reference}

S1. Du, C. Y.; Zhao, T. S.; Liang, Z. X. Sulfonation of Carbon-Nanotube Supported Platinum Catalysts for Polymer Electrolyte Fuel Cells J. Power Sources 2008, 176, 9-15.

S2. Berber, M. R.; Hafez, I. H.; Fujigaya, T.; Nakashima, N. Durability Analysis of Polymer-Coated

Pristine Carbon Nanotube-Based Fuel Cell Electrocatalysts under Non-Humidified Conditions $J$. Mater. Chem. A, 2014, 2, 19053-19059. 